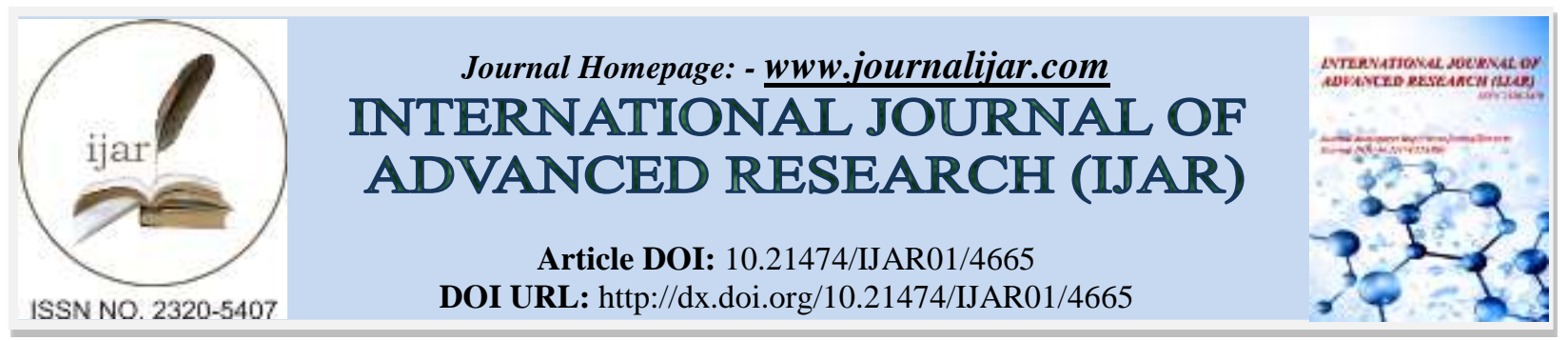

RESEARCH ARTICLE

\title{
SOME RANDOM FIXED POINT THEOREMS FOR SELF MAPPING IN BANACH SPACE.
}

Suhas S. Patil ${ }^{1}$ and U. P. Dolhare ${ }^{2}$.

1. Research Scholar, Science College (RC), SRTM University, Nanded (M.S), India.

2. Associate Professor, Department of Mathematics, D.S.M. College, Jintur (M.S), India.

\section{Manuscript Info}

Manuscript History

Received: 26 April 2017

Final Accepted: 30 May 2017

Published: June 2017

Key words:-

Banach Space, Self-Mapping, Fixed Points,

Contraction Mapping

\section{Abstract}

In this paper we establish some random fixed point theorems in Banach Space by new rational expression for Self Mappings which satisfy some contractive conditions.

\section{Introduction:-}

Banach Fixed Point theorem also called contraction mapping principle or contraction mapping theorem [5]. In metric space gives guarantees the existence and uniqueness of fixed point of some self-mappings of metric space providing constructive method stated by Stephan Banach in 1922. In recent years lot of work have been done in nonlinear analysis, the study of non-contraction mapping with the existence of fixed point take attention of some authors in non-linear analysis with the details of existence of a fixed point and also the non-expansive mapping.

Random fixed point theorems in abstract space are useful in the study of non-linear random equations for proving the existence and uniqueness of theorems. It's well known that a physical problems the differential and integral equations are generally non-linear, so Banach contraction principle [7] provides a powerful tool for getting the solution of their equation. Many problems of analysis and applied mathematics are used to find the solutions of nonlinear functional equations which can be formulated in terms of finding the fixed points of a non-linear mapping.

\section{Preliminaries:-}

We recall some definitions and properties of normed linear space.

Definition 2.1 A set X of elements is called a vector space or linear space or Linear Vector Space over the real's if we have a function + on $\mathrm{X} x \mathrm{X}$ to $\mathrm{X}$ and a function $\operatorname{dot}(\cdot)$ on $\mathrm{R} \mathrm{x} \mathrm{X}$ to $\mathrm{X}$ that satisfy the following conditions

- $x+y=y+x$

- $\quad(x+y)+z=x+(y+x)$

- There exist $\theta \in x$ such that $x+\theta=x$ for all $x \in X$.

- $\lambda(x+y)=\lambda x+\lambda y, \lambda \in R, x, y \in R$.

- $\lambda(\mu x)=(\lambda \mu) x, \lambda, \mu \in R, x \in X$.

- $\quad 0 . x=\theta, 1 . x=x$.

Here we call '+' addition and '.' scalar multiplication and $\theta$ is unique. 
Definition 2.2 Let $\mathrm{X}$ be a vector space over the real or complex number, A mapping $\|\|:. X \rightarrow R^{+}$is called a norm provided that the following conditions are satisfied following conditions

1. $\|x\|=0 \Leftrightarrow x=0$

2. $\|x+y\| \leq\|x\|+\|y\|$

3. $\|\alpha x\|=|\alpha|\|x\|$

If $\mathrm{X}$ is a vector space and $\|$.$\| is a norm on \mathrm{X}$ then the $\operatorname{pair}(x,\|\|$.$) is called norms vector space. We called \mathrm{X}$ is a metric space if $\mathrm{X}$ is a vector space and $\|$.$\| is norm on \mathrm{X}$ and we define metric $\mathrm{d}$ by $d(x, y)=\|x-y\|$ for all $\mathrm{x}, \mathrm{y}$ in $\mathrm{X}$.

If a normed vector space is complete in this metric then it is called a Banach Space.

Remark 2.1 If we define a metric space $\rho$ by $\rho(x, y)=\|x-y\|$ then a normed vector space becomes a metric space.

Definition 2.3 (Banach Space) A Banach Space $(x,\|\|$.$) is a normed vector space such that \mathrm{X}$ is complete under the metric included by the norm $\|$.

Definition 2.4 A sequence $\left\{x_{k}\right\}$ in a normed linear space is said to be a Cauchy sequence if $\left\|x_{k}-x_{l}\right\| \rightarrow 0$ as $k, l$ tends to infinity. i.e for given $\delta>0$ there exist an integer $\mathrm{N}$ such that $\left\|x_{k}-x_{l}\right\|<\delta$ for all $k, l>\mathrm{N}$.

Definition 2.5[9] Let $\mathrm{X}$ is a metric space equipped with a distance $\mathrm{d}$ and a mapping $\mathrm{f}$ from $\mathrm{X}$ to $\mathrm{X}$ is said to be Lipschitz continuous if there exist $\lambda \geq 0$ such that,

$$
d(f(k), f(l)) \leq \lambda d(k, l) \text { for all } k, l \in X .
$$

The $\lambda$ for which the above inequality holds is the Lipschitz constant of $f$.

If $\lambda=1$ then $\mathrm{f}$ is said to be non-expansive and if $\lambda<1$ then $\mathrm{f}$ is said to be a contradiction.

Fixed Point Theorem For Self Mapping In Banach Space:-

The Banach Fixed point theorem states as follows

Theorem 3.1[7] Let (X, $\delta$ ) complete metric space and $f: X \rightarrow X$ is a contraction then $\mathrm{f}$ has a unique fixed point. Theorem 3.2 Let $\mathrm{f}$ be mapping of a Banach space $\mathrm{X}$ into itself, if $\mathrm{f}$ satisfies the following conditions, $\mathrm{f}^{2}=\mathrm{I}$ where $\mathrm{I}$ is identity mapping.

$$
\begin{aligned}
&\|f(k)-f(l)\| \leq \alpha \frac{\|k-l\|\|k-f(k)\|+\|k-f(k)\|\|k-f(l)\|+\|k-f(l)\|\|l-f(k)\|}{\|k-l\|+\|l-f(l)\|} \\
&+\beta(\|k-f(k)\|+\|l-f(l)\|)+\delta(\|k-f(l)\|+\|l-f(k)\|)+\eta\|k-l\|
\end{aligned}
$$

Then for every $k, l$ belongs to $\mathrm{X}, 0<\alpha, \beta, \delta \& \eta<1$ and $5 \alpha+4 \beta+2 \delta+\eta$ is less than 2 then $\mathrm{f}$ has a fixed point. If $\alpha+2 \delta+\eta<1$ then $f$ has a unique fixed point.

Proof: Suppose that $k$ is a fixed point of the Banach Space $X$.

$$
\begin{aligned}
& \text { Let } \frac{(f+I) k}{2}, \quad m=f(l) \text { and } t=2 l-m \text { then we have } \\
& \|m-k\|=\left\|f(l)-f^{2}(k)\right\|=\| f(l)-f(f(k) \|
\end{aligned}
$$




$$
\begin{aligned}
& \leq \alpha \frac{\|l-f(k)\|\|l-f(l)\|+\|l-f(l)\|\left\|l-f^{2}(k)\right\|+\left\|l-f^{2}(k)\right\|\|f(k)-f(l)\|}{\|l-f(k)\|+\left\|f(k)-f^{2}(k)\right\|} \\
& +\beta\left(\|l-f(l)\|+\left\|f(k)-f^{2}(k)\right\|\right)+\delta\left(\left\|l-f^{2}(k)\right\|+\|f(k)-f(l)\|\right)+\eta\|l-f(k)\| \\
& \|m-k\| \leq \alpha \frac{\|l-f(k)\|\|l-f(l)\|+\|l-f(l)\|\|k-l\|+\|k-l\|\|f(k)-f(l)\|}{\|l-f(k)\|+\|k-f(k)\|} \\
& +\beta(\|l-f(l)\|+\|k-f(k)\|)+\delta(\|k-l\|+\|f(k)-f(l)\|)+\eta\|l-f(k)\| \\
& \|m-k\| \leq \alpha \frac{\|l-f(l)\|\|k-f(k)\|}{\|k-l\|}+\|f(k)-f(l)\| \\
& +\beta(\|l-f(l)\|+\|k-f(k)\|)+\delta(\|k-l\|+\|f(k)-f(l)\|)+\eta\|l-f(k)\| \\
& \|m-k\| \leq \alpha \frac{\|l-f(l)\|\|k-f(k)\|+\|k-l\|\|f(k)-f(l)\|}{\|k-l\|} \\
& +\beta(\|l-f(l)\|+\|k-f(k)\|)+\delta(\|k-l\|+\|f(k)-f(l)\|)+\eta\|l-f(k)\| \\
& \|m-k\| \leq \alpha \frac{\|l-f(l)\|\|k-f(k)\|}{\|k-l\|}+\|f(k)-f(l)\| \\
& +\beta(\|l-f(l)\|+\|k-f(k)\|)+\delta(\|k-l\|+\|f(k)-f(l)\|)+\eta\|l-f(k)\| \\
& \|m-k\| \leq \alpha\left[\frac{\|l-f(l)\|\|k-f(k)\|}{\left\|k-\frac{1}{2}(f+I) x\right\|}+\left\|f(k)-f\left(\frac{1}{2}(f+I) x\right)\right\|\right] \\
& +\beta(\|l-f(l)\|+\|k-f(k)\|)+\delta\left[\left\|k-\frac{1}{2}(f+I) x\right\|+\left\|f(k)-f\left(\frac{1}{2}(f+I) x\right)\right\|\right]+\eta\left\|\frac{1}{2}(f+I) x-f(k)\right\| \\
& \|m-k\| \leq \alpha\left[\frac{\|l-f(l)\|\|k-f(k)\|}{\frac{1}{2}\|k-f(x)\|}+\frac{1}{2}\|k-f(k)\|\right] \\
& +\beta(\|l-f(l)\|+\|k-f(k)\|)+\delta\left[\frac{1}{2}\|k-f(k)\|+\frac{1}{2}\|k-f(k)\|\right]+\eta \frac{1}{2}\|k-f(k)\| \\
& \|m-k\| \leq \alpha\left[2\|l-f(l)\|+\frac{1}{2}\|k-f(k)\|\right]+\beta \frac{1}{2}\|k-f(k)\| \\
& +\beta(\|l-f(l)\|+\|k-f(k)\|)+\delta[\|k-f(k)\|]+\eta \frac{1}{2}\|k-f(k)\| \\
& \|m-k\| \leq\left(\frac{\alpha}{2}+\beta+\delta+\frac{\eta}{2}\right)\|k-f(k)\|+(2 \alpha+\beta)\|l-f(l)\| \\
& \|t-k\|=\|2 l-t-k\|=\left\|\frac{1}{2}(f+I) x-m-x\right\|=\|f(k)-m\|=\|f(k)-f(l)\| \\
& \|m-k\| \leq \alpha \frac{\|k-l\|\|k-f(k)\|+\|k-f(k)\|\|k-f(l)\|+\|k-f(l)\|\|l-f(k)\|}{\|k-l\|+\|l-f(l)\|} \\
& +\beta(\|k-f(k)\|+\|l-f(l)\|)+\delta(\|k-f(l)\|+\|l-f(k)\|)+\eta\|k-l\| \\
& \|m-k\| \leq \alpha \frac{\|k-f(k)\|(\|k-l\|+\|k-f(k)\|)+\|k-f(l)\|\|l-f(k)\|}{\|k-f(l)\|}
\end{aligned}
$$




$$
\begin{aligned}
& +\beta(\|k-f(k)\|+\|l-f(l)\|)+\delta(\|k-f(l)\|+\|l-f(k)\|)+\eta\|k-l\| \\
& \|m-k\| \leq \alpha\left[\frac{\|k-f(k)\|\|l-f(l)\|}{\|k-f(l)\|}+\|l-f(k)\|\right] \\
& +\beta(\|k-f(k)\|+\|l-f(l)\|)+\delta(\|k-f(l)\|+\|l-f(k)\|)+\eta\|k-l\| \\
& \|m-k\| \leq \alpha\left[\frac{\|k-f(k)\|\|l-f(l)\|}{\| k-f\left(\frac{1}{2}(f+I) x \|\right.}+\left\|\frac{1}{2}(f+I) x l-f(k)\right\|\right] \\
& +\beta(\|k-f(k)\|+\|l-f(l)\|)+\delta\left(\left\|k-f\left(\frac{1}{2}(f+I) x\|+\| \frac{1}{2}(f+I) x-f(k) \|\right)+\eta\right\| k-\frac{1}{2}(f+I) x\|\| m-k \|\right. \\
& \|m-k\| \leq \alpha\left[\frac{\|k-f(k)\|\|l-f(l)\|}{\frac{1}{2}\|k-f(x)\|}+\frac{1}{2}\|k-f(k)\|\right] \\
& +\beta(\|k-f(k)\|+\|l-f(l)\|)+\delta\left(\frac{1}{2}\|k-f(k)\|+\frac{1}{2}\|k-f(k)\|\right)+\eta \frac{1}{2}\|k-f(k)\| \\
& \|m-k\| \leq \alpha\left[2\|l-f(l)\|+\frac{1}{2}\|k-f(k)\|\right]+\beta(\|k-f(k)\|+\|l-f(l)\|)+ \\
& +\delta(\|k-f(k)\|)+\eta \frac{1}{2}\|k-f(k)\| \\
& \|m-k\| \leq\left[\left(\frac{\alpha}{2}+\beta+\delta+\frac{\eta}{2}\right)\|k-f(k)\|+(2 \alpha+\beta)\|l-f(l)\|\right]
\end{aligned}
$$

Then,

$$
\begin{aligned}
\|m-t\| \leq & \|(m-k)-(k-t)\| \leq(\|(m-k)\|+\|(k-t)\| \\
& +\left[\left(\frac{\alpha}{2}+\beta+\delta+\frac{\eta}{2}\right)\|k-f(k)\|+(2 \alpha+\beta)\|l-f(l)\|\right] \\
+ & {\left[\left(\frac{\alpha}{2}+\beta+\delta+\frac{\eta}{2}\right)\|k-f(k)\|+(2 \alpha+\beta)\|l-f(l)\|\right] } \\
\|m-t\| \leq & {[(\alpha+2 \beta+2 \delta+\eta)\|k-f(k)\|+(4 \alpha+2 \beta)\|l-f(l)\|] }
\end{aligned}
$$

And also,

$$
\begin{aligned}
& \begin{aligned}
\|m-k\|=\|f(l)-(2 l-m)\| \\
=\| f(l)-2 l-f(l)) \| \\
=2\|l-f(l)\|
\end{aligned} \\
& \begin{aligned}
2\|l-f(l)\| \leq( & \alpha+\chi+2 \lambda+2 \mu+2 \beta+2 \delta+\eta)\|k-f(k)\|+(4 \alpha+2 \lambda+2 \beta)\|l-f(l)\| \\
& (2-(4 \alpha+2 \beta+2 \lambda))\|l-f(l)\| \\
\leq & {[(\alpha+\chi+2 \lambda+2 \mu+2 \beta+2 \delta+\eta)]\|k-f(k)\| }
\end{aligned} \\
& \|l-f(l)\| \leq \psi\|k-f(k)\|, \text { where } \quad \psi=\frac{(\alpha+\chi+2 \lambda+2 \mu+2 \beta+2 \delta+\eta)}{[2-(4 \alpha+2 \lambda+2 \chi]}<1
\end{aligned}
$$

Where, $5 \alpha+4 \beta+2 \delta+\eta<2$

Let us consider $\sigma=\frac{1}{2}(f+I)$ then for every $k \in X$, we have

$$
\begin{aligned}
\left\|\sigma^{2}(k)-\sigma(k)\right\| & =\|\sigma(l)-l\| \\
=\left\|\frac{1}{2}(f+I) l-l\right\| & \\
& =\frac{1}{2}\|l-f(l)\|
\end{aligned}
$$




$$
\leq \frac{\psi}{2}\|k-f(k)\|
$$

Then from the definition of $\psi$ we say that $\left\{\sigma^{2}(k)\right\}$ is a Cauchy sequence in $X$. And hence by completeness $\left\{\sigma^{2}(k)\right\}$ converges to some elements $k_{0} \in X$.

Hence,

$$
\begin{aligned}
& \lim _{n \rightarrow \infty} \sigma^{2}(k)=k_{0} \\
& \therefore \sigma\left(k_{0}\right)=k_{0}
\end{aligned}
$$

i.e. $k_{0}$ is fixed point of $f$.

About Uniqueness if possible let $l_{0} \neq k_{0}$ is another fixed point of $f$, then

$$
\begin{aligned}
& \left\|k_{0}-l_{0}\right\|=\left\|f\left(k_{0}\right)-f\left(l_{0}\right)\right\| \\
& \leq \alpha \frac{\left\|k_{0}-l_{0}\right\|\left\|k_{0}-f\left(k_{0}\right)\right\|+\left\|k_{0}-f\left(k_{0}\right)\right\|\left\|k_{0}-f\left(l_{0}\right)\right\|+\left\|k_{0}-f\left(l_{0}\right)\right\|\left\|l_{0}-f\left(k_{0}\right)\right\|}{\left\|k_{0}-l_{0}\right\|+\left\|l_{0}-f\left(l_{0}\right)\right\|} \\
& +\beta\left(\left\|k_{0}-f\left(k_{0}\right)\right\|+\left\|l_{0}-f\left(l_{0}\right)\right\|\right)+\delta\left(\left\|k_{0}-f\left(l_{0}\right)\right\|+\left\|l_{0}-f\left(k_{0}\right)\right\|\right)+\eta\left\|k_{0}-l_{0}\right\| \\
& \left\|k_{0}-l_{0}\right\| \\
& \leq \alpha \frac{\left\|k_{0}-l_{0}\right\|^{2}}{\left\|k_{0}-l_{0}\right\|}+2 \delta\left\|k_{0}-l_{0}\right\|+\eta\left\|k_{0}-l_{0}\right\| \\
& \leq \alpha\left\|k_{0}-l_{0}\right\|+2 \delta\left\|k_{0}-l_{0}\right\|+\eta\left\|k_{0}-l_{0}\right\| \\
& \leq \alpha\left\|k_{0}-l_{0}\right\|+2 \delta\left\|k_{0}-l_{0}\right\|+\eta\left\|k_{0}-l_{0}\right\| \\
& \left\|k_{0}-l_{0}\right\|=(\alpha+2 \delta+\eta)\left\|k_{0}-l_{0}\right\|
\end{aligned}
$$

This is a contradiction.

Hence $k_{0}=l_{0} \quad \therefore$ fixed point is unique.

This completes the proof.

Theorem 3.3[8] Let $\mathrm{f}$ be mapping of a Banach space $\mathrm{X}$ into itself, if $\mathrm{f}$ satisfies the following conditions,

$$
\begin{gathered}
\|f q-f(f p)\| \leq \alpha \frac{\|q-f q\|\|f p-p\|\|q-p\|+\|q-f p\|^{3}}{\|q-f p\|^{2}}+\beta \frac{\|f p-p\|\|f p-f q\|\|q-p\|+\|q-f p\|^{3}}{\|q-f p\|^{2}} \\
+\gamma[\|q-f q\|+\|f p-p\|]+\delta[\|q-p\|+\|f p-f q\|]+\eta[\|q-f p\|] \\
\leq \alpha \frac{\|q-f q\|\|f p-p\| \frac{1}{2}\|p-f p\|+\frac{1}{8}\|p-f p\|^{3}}{\frac{1}{4}\|p-f p\|^{2}}+\beta \frac{\|f p-p\|(\|f p-q\|+\|q-f q\|) \frac{1}{2}\|p-f p\|+\|q-f p\|^{3}}{\frac{1}{4}\|p-f p\|^{2}} \\
+\gamma[\|q-f q\|+\|f p-p\|]+\delta\left[\frac{1}{2}\|p-f p\|+\|f p-q\|+\|q-f q\|\right]+\eta \frac{1}{2}[\|p-f p\|]
\end{gathered}
$$

$\mathrm{f}^{2}=\mathrm{I}$, where I is identity mapping

$$
\begin{aligned}
&\|f p-f q\| \leq \alpha \frac{\|q-f q\|\|p-f p\|\|p-f q\|+\|p-q\|^{3}}{\|p-q\|^{2}}+\beta \frac{\|q-f q\|\|q-f p\|\|p-f q\|+\|p-q\|^{3}}{\|p-q\|^{2}} \\
&+\gamma[\|p-f p\|+\|q-f q\|]+\delta[\|p-f q\|+\|q-f p\|]+\eta[\|p-q\|]
\end{aligned}
$$

With the equation $10 \alpha+9 \beta+8 \gamma+5 \delta+\eta<4$ and $p \neq q$, then it has a unique fixed point.

Theorem 3.3[6] Let $\mathrm{f}$ be mapping of a Banach space $\mathrm{X}$ into itself, if $\mathrm{f}$ satisfies the following conditions, $\mathrm{f}^{2}=\mathrm{I}$, where I is identity mapping then, 


$$
\begin{aligned}
\| f p-f q) \| \leq \alpha \max & {\left[(\|p-q\|),\|p-f p\|,\|q-f q\|, \frac{\|p-f p\|\|q-f q\|}{1+\|p-q\|}\right] } \\
+\beta & {[\|p-f p\|+\|q-f q\|]+\gamma[\|p-f q\|+\|q-f p\|]+\delta[\|p-q\|] }
\end{aligned}
$$

Then for every $p, q$ belongs to $\mathrm{X}, 0<\alpha, \beta, \gamma \& \delta<1$ and $4 \beta+3 \gamma+3 \alpha+\delta<2$ is less than 2 then $\mathrm{f}$ has a fixed point. If $\alpha+2 \gamma+\delta<1$ then $f$ has a unique fixed point.

\section{Conclusion:-}

In this paper we have presented some random fixed point theorems by new rational expression for Self Mappings in Banach Space which satisfy some contractive conditions

\section{Acknowledgements:-}

The author thanks an anonymous referee for his valuable suggestions that helped to improve the final version of this paper.

\section{References:-}

1. M. S. Khan, "Some Fixed Points Theorems In Metric and Banach Space, Indian Jou. Pure Appl.Math.,11(4) (1980) 413-421.

2. M. S. Khan And M. Swaleh, "Fixed Point Theorems For Generalized Contraction, Indian Jou. Pure Appl. Math., 15(9) (1984) 984-990.

3. T. H. Chang And C. H. Yen, "Some Fixed Point Theorems In Banach Space", Jou. of Math Anal. \& Appl. 138(1989) 550-558.

4. B. K. Ray And S. P. Singh, "Fixed Point Theorems In Banach Spaces", Int. J. Math \& Math.Sci, Vol-9(4) (1986) 771-779.

5. Suhas S. Patil \& Uttam P. Dolhare, “Some Common Fixed Point Theorems In Complete Metric Space Via Weakly Commuting Mappings", International Jou. Appl. Pure Science \& Agriculture", Vol-2(10) (2016) 3238.

6. P. Bhatnagar, A. Tenguriya, R. N. Yadava, "Some Common Fixed Point Theorems In Banach Space For Self Mappings", South Asian Jou. Math., Vol.2 (3) (2012) 242-247.

7. Suhas S.Patil \& U. P. Dolhare,“A Note On Development Of Metric Fixed Point Theory”, Int. Jou. Adv. Reserch", Vol-4(8) (2016) 1729-1734.

8. R. Shrivastava, J. Singhvi, R. Bhardwaj And S. Patkar, Some Fixed Point And Common Fixed Point Theorems In Banach Spaces For Rational Expression”, Int. Jou. Of Theoritical And Appl. Sci. 3(2) (2011) 107115.

9. Suhas Patil, Uttam. Dolhare, "Random Fixed Point Theorems For Contraction Mappings In Metric Space", Int. Jou. Of Sci. \& Res., Vol-5(10) (2016) 1172-1176.

10. R. Bhardwaj, B. Wadkar, B. Singh, "Fixed Point Theorems in Generalized Banach Space", Int. Jou. Of Comp. \& Math. Sci., Vol-4 (2015) 96-102.

11. Suhas Shivajirao Patil, Uttam Prallahadrao Dolhare,"Random Fixed Point Theorems For Multi-Valued Contraction Mapping In Complete Metric Space”, Int. Jou. of Current Research., Vol-12 (2016) 42856-42860

12. Kirk W. A, "Fixed Point Theorems for non-expansive mappings", Contem Math.18 (1983) 121-140.

13. Suhas Shivajirao Patil, U. P. Dolhare," Random Fixed Point Theorems For Compatible Mappings In Metric Space”, International Conference on Mathematical Analysis and its Applications (ICMAA, March 5-9, 2017) held in Dayanand Science College, Latur-413512( Maharashtra. _) 